\title{
The patient's perspective on blue light flexible cystoscopy: insight from a prospective clinical study
}

\author{
Nima Almassi, Eugene Pietzak \\ Urology Service, Department of Surgery, Memorial Sloan Kettering Cancer Center, New York, NY, USA \\ Correspondence to: Eugene Pietzak, MD. Urology Service, Department of Surgery, Memorial Sloan Kettering Cancer Center, 1275 York Avenue, New \\ York, NY, 10065, USA. Email: pietzake@mskcc.org. \\ Provenance: This is an invited Editorial commissioned by Section Editor Xiao Li (Department of Urology, Jiangsu Cancer Hospital \& Jiangsu \\ Institute of Cancer Research \& Nanjing Medical University Affiliated Cancer Hospital, Nanjing, China). \\ Comment on: Smith AB, Daneshmand S, Patel S, et al. Patient-reported outcomes of blue-light flexible cystoscopy with hexaminolevulinate in the \\ surveillance of bladder cancer: results from a prospective multicentre study. BJU Int 2018. [Epub ahead of print].
}

Submitted Sep 12, 2018. Accepted for publication Sep 18, 2018.

doi: $10.21037 /$ tau.2018.09.10

View this article at: http://dx.doi.org/10.21037/tau.2018.09.10

Patient-reported outcomes (PRO) are recognized as an increasingly important area of investigation in cancer studies, encompassing a broad range of measures assessing patient anxiety, pain, satisfaction, and quality of life. The importance of incorporating PRO in clinical studies is highlighted by the low concordance between the treating clinician's perception and the patient's self-reported experience of symptoms and treatment-related adverse events (1-3). Understanding the physical, emotional, and psychosocial impact of different treatment modalities on the patient's experience and quality of life has important implications for quality improvement, patient counseling and treatment selection in a patient-centered model. Bladder cancer patients have among the lowest self-reported satisfaction in treatment-related decision-making relative to other cancer patients (4), yet a significant proportion of randomized clinical trials in bladder cancer failed to include PRO measures (5), emphasizing this unmet need.

Daneshmand and colleagues recently reported the results of a phase III trial comparing blue light flexible cystoscopy (BLFC) and white light flexible cystoscopy in the detection of bladder cancer among patients undergoing surveillance cystoscopy (6). BLFC was demonstrated to improve detection of recurrent bladder tumors, with 20.6\% of patients with recurrent tumors diagnosed only on BLFC. Additionally, among patients with recurrent disease, blue light cystoscopy identified additional malignant lesions in $46 \%$ of patients. These investigators incorporated PRO measures in the trial design, allowing prospective study of satisfaction, anxiety, and willingness to pay among patients undergoing blue light flexible cystoscopy, the results of which were recently published by Smith and colleagues in BJU International (7). In this trial, all patients were administered questionnaires to assess anxiety and pain using validated questionnaires at the initial screening office visit and again immediately following surveillance BLFC. For patients with abnormal surveillance cystoscopy who were referred to the operating room for biopsy or transurethral resection, the questionnaires were again administered after the patient had received the pathology results. A separate questionnaire was administered to assess patient perspective on whether BLFC "was worth it" and whether patients would be willing to pay out of pocket for this procedure.

The investigators report a 2.6-point decrease in the mean anxiety score following BLFC relative to the initial screening visit, a magnitude of change that has been previously-reported to be clinically meaningful (8). Not surprisingly, a greater decrease in mean anxiety score was observed in patients who had a negative surveillance cystoscopy (-3.5 vs. -1.2 points). Among patients referred to the operating room for biopsy or transurethral resection, a further 3.4-point decrease in mean anxiety score was observed for patients with negative pathology results, whereas patients with positive pathology results demonstrated no change in mean anxiety score. Over $90 \%$ of patients perceived BLFC to be worthwhile irrespective of BLFC findings (normal vs abnormal) or BLFC performance (true positive $v s$. false positive), with few patients reporting 
a diminished quality of life after BLFC (1\%) or reporting the experience to be worse than expected (2\%).

There are a few important considerations when interpreting these findings. First, all patients underwent intravesical hexaminolevulinate instillation before surveillance cystoscopy, with PRO measures compared before and after BLFC. Without a control group against which these PRO measures can be compared, it remains unknown whether the additional preprocedural catheterization, intravesical instillation, and the 1-3 hours wait before undergoing BLFC impacts patient anxiety relative to patients undergoing standard white light surveillance cystoscopy or narrow-band imaging (NBI) flexible cystoscopy. Importantly, BLFC did not result in higher anxiety scores and did not appear to significantly increase patient-reported pain. A high percentage of patients reported a positive perception of BLFC irrespective of the test's performance, perhaps a result of selection bias reflecting the baseline perception of patients willing to participate in such a trial. Although the majority of patients reported a willingness to pay, this metric has been shown to correlate poorly with actual patient behavior and should be interpreted with caution (9). Ultimately, to truly know whether BLFC "was worth it", its clinical utility will need to be prospectively determined by improved recurrence-free and progression-free survival outcomes compared to WLC and other "enhanced" cystoscopy techniques, such as NBI.

Overall, these findings provide important insight into the patient-reported experience with BLFC that will be valuable when counseling bladder cancer patients undergoing surveillance cystoscopy. Smith and colleagues should be commended for incorporating PRO measures when designing this phase III clinical trial. As advances in the detection and treatment of bladder cancer are realized, it remains imperative to examine and understand the physical, emotional, and psychosocial impact these interventions have on the bladder cancer patient. The insights gained will drive improvement in the quality and value of care provided to our patients.

\section{Acknowledgements}

Funding: This work was supported by the Sidney Kimmel Center for Prostate and Urologic Cancers, the Michael and Zena Wiener for Therapeutics Program in Bladder Cancer, Pin Down Bladder Cancer, and the National Cancer Institute Cancer Center Core Grant Number P30-CA008748.

\section{Footnote}

Conflicts of Interest: Dr. Pietzak is on a Scientific Advisory
Board for Merck. Dr. Almassi has no conflicts of interest to declare.

\section{References}

1. Gravis G, Marino P, Joly F, et al. Patients' self-assessment versus investigators' evaluation in a phase III trial in noncastrate metastatic prostate cancer (GETUG-AFU 15). Eur J Cancer 2014;50:953-62.

2. Camuso N, Bajaj P, Dudgeon D, et al. Engaging Patients as Partners in Developing Patient-Reported Outcome Measures in Cancer-A Review of the Literature. Support Care Cancer 2016;24:3543-9.

3. Macquart-Moulin G, Viens P, Bouscary ML, et al. Discordance between physicians' estimations and breast cancer patients' self-assessment of side-effects of chemotherapy: an issue for quality of care. Br J Cancer 1997;76:1640-5.

4. El Turabi A, Abel GA, Roland M, et al. Variation in reported experience of involvement in cancer treatment decision making: evidence from the National Cancer Patient Experience Survey. Br J Cancer 2013;109:780-7.

5. Feuerstein MA, Jacobs M, Piciocchi A, et al. Quality of life and symptom assessment in randomized clinical trials of bladder cancer: A systematic review. Urol Oncol 2015;33:331.e17-23.

6. Daneshmand S, Patel S, Lotan Y, et al. Efficacy and Safety of Blue Light Flexible Cystoscopy with Hexaminolevulinate in the Surveillance of Bladder Cancer: A Phase III, Comparative, Multicenter Study. J Urol 2018;199:1158-65.

7. Smith AB, Daneshmand S, Patel S, et al. Patientreported outcomes of blue-light flexible cystoscopy with hexaminolevulinate in the surveillance of bladder cancer: results from a prospective multicentre study. BJU Int 2018. [Epub ahead of print].

8. Schalet BD, Pilkonis PA, Yu L, et al. Clinical validity of PROMIS Depression, Anxiety, and Anger across diverse clinical samples. J Clin Epidemiol 2016;73:119-27.

9. Vernazza CR, Wildman JR, Steele JG, et al. Factors affecting patient valuations of caries prevention: Using and validating the willingness to pay method. J Dent 2015;43:981-8.

Cite this article as: Almassi N, Pietzak E. The patient's perspective on blue light flexible cystoscopy: insight from a prospective clinical study. Transl Androl Urol 2018;7(Suppl 6):S727-S728. doi: 10.21037/tau.2018.09.10 